\title{
SELECTED ISSUES OF IP TELEPHONY
}

Technologies for transporting voice over networks based on IP protocol, known under various names, e.g., Voice Over IP (VoIP), Voice on the Net (VoN), IP telephony (below IP telephony), made a great progress in the last decade. IP telephony has become one of the most important and the most rapidly developing Internet technologies that is offering a technical as well as economical alternative to the existing telecommunication networks. The paper points three issues that from the authors' point of view seem to be crucial for a wide deployment of IP telephony in European networks: Development and implementation of new IP telephony services based on the SIP (Session Initiation Protocol), Interworking between IP-based network signalling protocols and signalling protocols used in traditional SCNs (Switched Communication Networks) and the quality of voice transmission over IP networks. The paper also gives some examples of practical solutions of the problems mentioned above.

\section{Introduction}

Technologies for transporting voice over networks based on IP protocol, known under various names, e.g., Voice Over IP (VoIP), Voice on the Net (VoN), IP telephony (below IP telephony), made a great progress in the last decade. IP telephony has become one of the most important and the most rapidly developing Internet technologies that is offering a technical as well as economical alternative to the existing telecommunication networks. Dynamics of the IP telephony development, successful handling of a standardisation process, increasing offer of IP telephony products, successful implementation of IP technologies, and especially, offer of new attractive communication services on the market, seem to be the key steps in a process of convergence of different services and networks on the IP technology basis.

IP telephony as a service does not mean only classical telephone services like we know them from classical circuit-switched networks (PSTN - Public Switched Telephone Network, ISDN Integrated Switched Telephone Network, GSM - Global System Mobile), but it offers also opportunities for creation of new attractive services based on integration of telecommunication and data worlds. In this "integrated, or converged world", other types of communication tools, apart from voice, may be used. This includes video, application sharing, virtual reality, etc.

Having taken into account the extreme opportunities of IP telephony for creation of new services, it is necessary to provide the right tools to speed up their development and implementation. There is a strong relation between the tools for service creation and a protocol that will be used for service delivery. In other words, the properties of a protocol have a strong impact on the opportunities for service creation.

In connection with new services provided over IP networks, the Session Initiation Protocol (SIP) is nowadays mentioned as the most perspective one. The main reason for that is its simplicity, the same principles with other Internet protocols (e.g., HTTP, SMTP) as well as the fact that it can be used as a control mechanism for multimedia multiparty connections, new types of services (e.g., Instant Messaging, etc.) as well as a signalling mechanism in Next Generation Networks (NGNs).

Despite the fact that there are some operators that are already offering IP telephony service based on the SIP (e.g., MCI in the USA, Telia in Europe), SIP technology still has to solve a number of issues before it can be widely deployed in European networks. Among those that are very topical, are: resources for the right tools for the development of new services that would advance the service of IP telephony at a qualitatively higher level (e.g., SIP http integration), possibilities for interworking of existing network types and terminals with IP-based networks and new types of terminals for IP telephony, as well as the Quality of Service (QoS) for IP telephony.

\section{Development and implementation of new IP telephony services based on the SIP protocol}

The importance of Internet within the world telecom industry has grown rapidly in several last years. There is evident renaissance of communications visible mainly in the Internet world and feasible thanks to a suite of new signalling and control protocols, which are based on completely different principles as the centralized control systems used in the traditional circuit-switched systems. SIP - Session Initiation Protocol, as a signalling protocol developed to set up, modify and tear down multimedia sessions over Internet. Thanks to it close relations to the HTTP and SMTP protocols, the traditional telecom signalling and control models for telephony could be moved towards the Internet and web-based protocols. SIP does not provide only seamless integration of telephony and conferencing with many other WWW and messaging applications,

\footnotetext{
* Martin Klimo, Tatiana Kováčiková, Pavol Segeč

Department of InfoCom Networks, Faculty of Management Science and Informatics, University of Žilina
} 
but benefits also from new forms of communications and new service features, like presence, instant messaging or mobility. Due to these facts SIP belong definitely among the key IP protocols.

In the Department of InfoCom Networks, the University Infoline (UI) service has been developed and implemented. The UI service integrates voice SIP and web services. This kind of services is related to the category of enhanced "click-to-call" services. The developed service is fully operational and the function of the service was tested in the framework of the international research project Eurescom P1111 NGOSSIP [1] through Internet. For the service purposes a SIP communication infrastructure has been built up at the Department of Information Networks.

\section{SIP (Session Initiation Protocol)}

SIP is a signalling protocol developed to set up, modify and tear down multimedia sessions over the Internet. SIP provides signalling and control functionality for a large range of multimedia communications. The main functions are: location of parties, invitation to service sessions, and negotiation of session parameters. To accomplish this, SIP uses a small number of text-based messages, which are exchanged between the SIP peer entities, i.e., SIP User Agents in user terminals. In time of messages exchange messages can traverse the network entities like proxy servers or redirect servers, which are used for support, like address resolution, routing calls to other entities, etc.

SIP only defines the initiation of a session; all other parts of the session are covered by other protocols. The session itself is then described in two levels. The SIP itself contains the parties' addresses and protocol processing features. The description of the media streams exchanged between the parties of a session is defined by another protocol. The Session Description Protocol (SDP) is used for this. The SDP defines text-based media description format that can be carried in the SIP message body. The message body is transparent to SIP; thus, any other session description can be carried (e.g., a weblink). From this point of view, SIP sessions are not limited to telephony calls or conferences only, but they can include any session description, for example web link, picture, email address.

\section{Programming SIP services and features}

For defining services, SIP took a different approach as compared to standard telephony. Implementation of SIP services can be done in general:

- Using SIP baseline protocol mechanisms

- Defining extensions (headers, methods-SIP call control framework)

- Dedicated programming tools (SIP-CPL, SIP-CGI, SIP-servlets, Java applets, JAIN APIs, Parlay).

From another point of view, SIP services can be programmed either by the trusted (such as administrators), or by the untrusted (such as end users) users.

The example of the UI service shows the implementation of SIP service by the use of Java programming language and the proprietary SIP API by the trusted users.
Independently on a programming tool, service creation has the meaning of the implementation of a service logic that guides behaviour of each of the system elements and controls a specific message flow or reacts on a message request. In the UI service, a service logic is implemented on the SIP application server (which assemblies the functions of the SIP registrar, SIP proxy, Third Party Call Control module) side. Thus, the service logic is a Java programme, which directs the application server's actions based on inputs from the SIP messages exchanged between SIP elements, the service logic and localization database and the data got from the Web page.

\section{Integration of web and SIP technologies}

The UI Service integrates dynamic web technologies (Java Server Pages, Java Applet) with SIP. This kind of web and SIP integration brings a new communication environment, which provides new communication features allowing to program a vast number of new services, from the traditional telephone service to advanced multimedia communication services. Such services may include, for example, click-to-dial services, SIP profile management through a web interface, presence services, etc., which provide the user web and SIP data integrated into a web page.

Due to a similar client-server nature of both technologies, integration of SIP and web technologies shows the following aspects.

A client side aspect is the first one. This includes adding SIP multimedia and signalling capabilities into the client side and integration of these capabilities together with web. It can be done through SIP integration into the client web software (i.e. browsers). Browsers become universal desktop tools with the multimedia capability. To achieve such effect, incorporation of some kind of a proprietary SIP enabled plug-ins into a browser technology, or in a case of Java UA (standalone SIP UA or lightweight Java Applet SIP UA) adding support for the JAVA technology to a client operating system, is required.

The second aspect is the integration of web server technology with SIP server technology. This can be done, for example, through some other technologies, e.g. through database sharing.

The last aspect resides in a design and supplying of the information flows between client sides and server entities involved in a service. For example the service UI is composed of web browsers, a web server, SIP UAs, SIP registrar and proxy servers, a database and the information flows among them.

\section{The service University Infoline}

There is no doubt, that WWW service is the most popular Internet service, and popularity and utilization of this service still grows rapidly. This has been enabled by the fast development of dynamic web technologies (for example JSP), which are changing browser technology to complex desktop tools. On the other hand, SIP is bringing new multimedia features to these communications environments.

The University Infoline service has been developed by the Department of Information Networks at the University of Zilina. The service integrates both web and SIP technologies. The service 
belongs to the group of click2call services and enables web browsing simultaneously with the establishment of SIP (Session Initiation Protocol) calls with persons who are responsible for a content of the web page (University / Educational Centre / Restaurant / Infoline etc.) The service is implemented as a part of distance learning environments of the departmental network, but in general, it may be implemented and used as any infoline service.

From a user point of view in the UI service, a student retrieves courses, which are listed in the web page of the University web space. Browsing a list of courses, a student wishes to get the detailed information on the course, provisions, etc. When he clicks on the hyperlink, a window with the course description appears together with the presence information of a tutor and a proposal to establish a call to a tutor of a course. If he accepts the offer, he becomes the SIP caller. The tutor becomes a callee.

Up to now, it looks more or less like a "classical click2call" scenario. In the frame of the service, we were interested in different states of a SIP call, which may occur between a student and a tutor and how to handle these states according to the tutors' personal preferences (system view). Under "tutors' personal preferences", we understand the possibility of a tutor to setup the time of day when he/she is available and when he/she wishes to accept calls initiated from the web on his/her personal SIP UA (User Agent). Thus, the following states of a tutor has to be distinguish and handle:
1. Tutor is online and available to accept calls initiated by a student from the web

2. Tutor is online, but busy with another call

3. Tutor is online, but not available to accept calls from the web

4. Tutor is offline.

In case 1., the SIP call between the tutor and the student is established.

In case 2. - 4., some options are made available for a student how to proceed. The options include providing a tutor contact addresses, i.e. e-mail address and SIP address of a called tutor, which can be used by a student to contact a tutor manually later and/or the possibilities, which allow a student to leave his/her contact addresses to the infoline system (e-mail or SIP address of a student, to which the system will sent the e-mail or establish a SIP call in the case a called tutor becomes online and available.

A complete service operates then in the following way (Fig. 1).

(1) Administrator creates a tutor account. (2) Data is recorded to a DB through JDBC. (3)(4) New tutor registers to his service web account through web and modifies his settings. (5) SIP registration of the tutor. (6) Registrar records the registration data to the DB. (7) Student is browsing through the list of courses and selects one of them. (7) Http request for a course is sent. (8) Web server loads SIP presence info of a tutor from DB. (9) Web page with course description and SIP presence sent back. In the case of

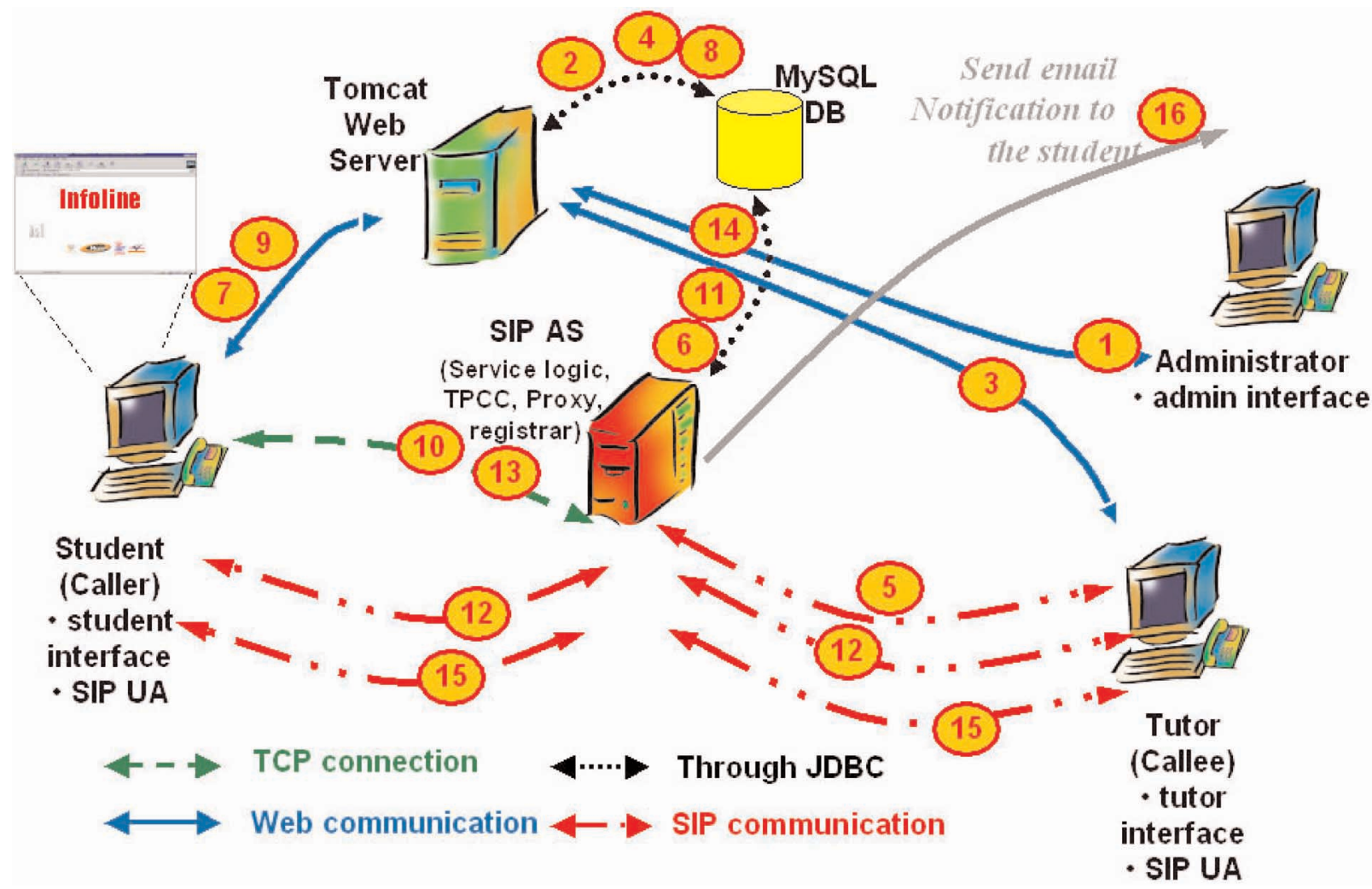

Fig. 1 Architecture of University Infoline service 
a "clicking" on the call button, the Java Applet is loaded in the same way. (10) Applet loading finished; the applet establishes socket connection to the SIP AS and initiates a SIP call. (11) Service logic tests tutor status. (12) Tutor is online and available; TPCC establishes a call. (13) Tutor is not available; student is leaving his contact addresses. (14) Service logic records the student contact data to the DB. (15) Tutor is becoming online and available - system establishes the communication based on the student contact addresses (16) And / or system sends e-mail to the student

The Service has been designed as hardware and operating system platforms independent. Therefore all the used server software components (database, web and SIP servers) are available for more OS platforms. The service logic has been developed using Java and the StarSIP API supported by the StarSIP application server. As a web technology has been used JSP together with JavaApplet technology.

Efficient programming of new communication services is a key issue for Internet telephony. With SIP, services can be created that combine elements from telephony and other web applications such as email, messaging, the Internet and video streaming. Using SIP, services like "click-to-call" become possible, in which, the user profiles can be managed through a web interface. On an example of the University Infoline service, we present a category of new enhanced "click-to-call" services, which allow a user browsing through the web pages not only to establish a SIP call, but which enable, via the service logic, to handle different states of a call based on the preferences of calling and called parties. The service is simply integrable to any type of voice-enhanced e-commerce services.

\section{Interworking between IP-based network signalling protocols and signalling protocols used in traditional SCNs (Switched Communication Networks)}

Historical operators have widely deployed SCN (or TDM) networks over decades for providing basic call services. With the popularity of the Internet and the low price of IP-oriented equipment, a new orientation for basic call has emerged: IP telephony. Even though SIP is not limited to IP telephony, SIP has raised big interest in telcos and telecom equipment manufacturers research and development laboratories. Two worlds for basic call now coexist: TDM networks and IP-oriented networks. If a subscriber in a world wants to communicate with a subscriber in the other world, the two worlds need to interwork.

For customers two kinds of SIP phones exist: soft-phone and hard-phone. Soft-phone runs on operating system (such as Windows $\mathrm{XP}$ ) of a computer connected to the Internet. Hard-phone implements SIP stacks on phone-like equipment that is connected to an Ethernet plug.

A customer using SIP phones cannot limit his phone calls to other SIP-based users, interest is in interworking with other telephone technology such as PSTN, GSM and other IP technology such as H.323. Anyhow final destination for a phone call cannot be pre-determined when using SIP technology (when interworking gateways are implemented in the network).

With the emergence of the Internet, a new kind of network architecture has appeared: Next Generation Networks (NGNs). A NGN is built on packet networks in order to transport both data and voice. The convergence of data and voice over the same typology of network (packet) will decrease investments by telcos. There are many definitions of NGN, however, two of the principal characteristics of the NGN are usually common:

- it is be based on a unique and shared packet-based network (IP, ATM, ...)

- transport layer and control layer are independent.

This brings also the necessity of the separation of control functions for bearer control, call control and service control functions and leads to:

- replacement of the traditional switching systems by new types of functional elements at the control layer

- appearance of new signalling protocols between different functional elements.

The full NGN architecture comprises network elements needed for the provision of traditional telephony services and advanced next-generation applications [Eurescom Project P1109: Next Generation Networks: the service offering standpoint]. They include Media Gateway, Signalling Gateway and Call Controller (Media Gateway Controller)

Media Gateway (MG) converts media and framing protocols provided in one type of network to the format required in another type of network. The Media Gateway terminates the bearer control protocols and contains bearer terminations. It also contains media manipulation equipment (e.g., transcoders, echo cancellers, or tone senders).

The MG should also provide a Signalling Gateway function when it terminates call signalling from access networks and has to relay it to the Call Server. The Media Gateway may therefore provide terminations such as Q.931 on the PSTN's side and IUA (ISDN User Adaptation layer) over SCTP/IP on the NGN side.

The Media stream policy/classification functions (e.g. control of traffic contracts: peak cell rate, average cell rate etc. in ATM, QoS class / priority management for Diffserv / RSVP in IP) are also provided by the Media Gateway.

Signalling Gateway (SG) is the network entity responsible to forward call control signalling by converting the transport mechanism of the incoming signalling to an appropriate ongoing transport mechanism (e.g., SS7 call signalling over MTP onto SS7 over IP).

In order to provide this function the Signalling Gateway may exist as a separate physical entity or reside within the same physical entity that support Media Gateway function depending on the 
particular network scenario. In the case that the Call server terminates call signalling from access network, the Signalling Gateway function is useless.

Call Server, or Media Gateway Controller (MGC) provides basic call control including call routing (routing tables, address translations between different numbering plan formats, routing information retrieval from external devices), call signalling process (SIP, H.323, ISUP, MGCP, etc.) and H.248-like Media Gateway Controller functions.

It should also provide more advanced call control functions like third party call control and CLASS services.

In addition, it must provide standard and open interfaces towards Application Servers to enable call related event triggering, service and policy control (e.g., personalized QoS policies, AAA policies, etc.).

In the NGN architecture, the Call Server presents the central node that supports the intelligence of communication.

Examples of Call servers are call agents, softswitches, SIP Server and H.323 gatekeepers.

Thanks to decomposition of the network functions into layers the physical implementation and geographical localization of the functional elements mentioned above is left on the choice of constructors and network operators. This also allows to optimize the network resources and to dimension them separately.

\section{Call control protocols}

Call control protocols allow the establishment, in general based on the user demand, the communication between two terminals or between a terminal and a server. The two candidate protocols are H.323 and SIP.

\section{Media Gateway control protocols}

The necessity to interconnect the traditional telephone networks into NGN as well as the flexibility allowed by the separation of the NGN transport and control layers have lead to the distinction of the functions of the Media Gateway and Call Server. This was the reason for developing a protocol, which allows the Call server to control the MG. In fact, it is a protocol, which allows for co-ordination between the transport and control layers. So far, two protocols for controlling the MG have been proposed: MGCP (Media Gateway Protocol) and MEGACO/H.248. These are relatively low-level device-control protocols that instruct an MG to connect streams coming from outside a packet network onto a packet stream such as the RTP (Real-Time Transport Protocol). Both protocols follow the master/slave approach based on the distribution of the network gateway functions into "more intelligent" (master) "and less-intelligent" (slave) parts. This approach enables centralization of application intelligence in relatively fewer control servers (MGCs in the MGCP and MEGACO/H.248 terminology) on the one hand and highly-cost and performance optimised gateway devices (MGs) on the other hand.

Signalling protocols between Call Servers (MGCS)

There are two types of signalling protocols that might be used between MGCs:
- at the level of a core network (BICC, SIP-T, H.323)

- for the interconnection with the existing PSTN/ISDN networks, via the transport of PSTN/ISDN signalling over an IP-based network (SIGTRAN).

The SIGTRAN (Signalling Transport) protocol suite was developed within the IETF to allow an interworking between SS7 network elements and IP-based elements. Its primary purpose is to address the transport of PSTN/ISDN signalling over IP networks, taking into account the functional and performance requirements of the PSTN/ISDN signalling. To interwork with the PSTN/ISDN, IP-based networks need to transport signalling such as DSS1 (Digital Subscriber System), or SS7 (e.g., ISUP, SCCP) messages between IP nodes such a SG (Signalling Gateway), a MGC (Media Gateway Controller), and a (MG) Media Gateway.

The architecture that was defined by SIGTRAN WG consists of three components:

- A standard IP

- A common signalling transport protocol - a protocol that supports a common set of reliable transport functions for signalling transport. A SCTP (Stream Control Transmission Protocol) has been defined for this purpose by the IETF and endorsed by the ETSI.

- An adaptation sub-layer that supports specific primitives, such as management indications, required by a particular signalling application protocol. The adaptation layers are different for different splits of the protocol. A number of different adaptation layers are being developed by the SIGTRAN group:

- IUA: The boundary is Q.921/Q.931 [2]

- M2UA: The boundary is MTP2/MTP3 [3]

- M2PA: The boundary is MTP2/MTP3, but for a symmetric scenario [4]

- M3UA: The boundary is MTP3/user part [5]

- SUA: The boundary is SCCP/SCCP user [6]

The adaptation layers mentioned above have been recently endorsed by the ETSI TC SPAN.

As mentioned above, the Session Initiation Protocol (SIP) is a "hot" candidate for being a call control protocol for new IP-oriented services. Even though SIP is not yet widely deployed in public networks, when this case occurs SIP needs to interwork with call control protocols in existing SCN networks such as ISUP, DSS1, or INAP. Within the EURESCOM project P1111 N-GOSSIP [1], the Department of InfoCom Networks from the University of Žilina was involved in, scenarios for SIP interworking with existing signalling protocols (ISUP, INAP, DSS1) were analysed.

Voice calls do not always have to originate and terminate in the PSTN (via MGCs). They may either originate and/or terminate in SIP phones. The alternatives for call origination and termination suggest the following possibilities for calls that traverse through an IP network:

1. PSTN origination - IP bridging - PSTN termination It is the situation in which a SIP network connects two instances of the telephone network. A telephone call originates in the PSTN and a SS7 ISUP message is dispatched to the MGC, 
which is the point of interconnection with the PSTN network. In this case, the MGC is the point of origination for message flows for this call over the IP network. The call is passed through proxies that route the call to the appropriate PSTN interface. The MGC that interconnects to the PSTN at the output of the IP network is the point of termination of the IP message flow. This MGC then uses ISUP to communicate with the PSTN at the terminating end.

The role of the SIP in the IP network is:

- to determine the appropriate point of termination

- to establish a session between the points of origination and termination in order to carry the call through the IP network.

2. PSTN origination - IP termination

This is a case when a call originates from the PSTN and terminates at a SIP phone.

3. IP origination - PSTN termination

A call originates from a SIP phone and terminates in the PSTN There is no telephony interface at call origination.

4. IP origination - IP termination: this is a case of pure SIP, with no PSTN involvement.

Thus, from a functional point of view, there are three distinct elements in a SIP VoIP network offering PSTN inter-connection:

- The originator of SIP signalling

- The terminator of SIP signalling

- The network of SIP proxies that routes calls from the originator to the terminator.

Based on this, the general SIP-ISUP interworking architecture was proposed as depicted in the following Figure:

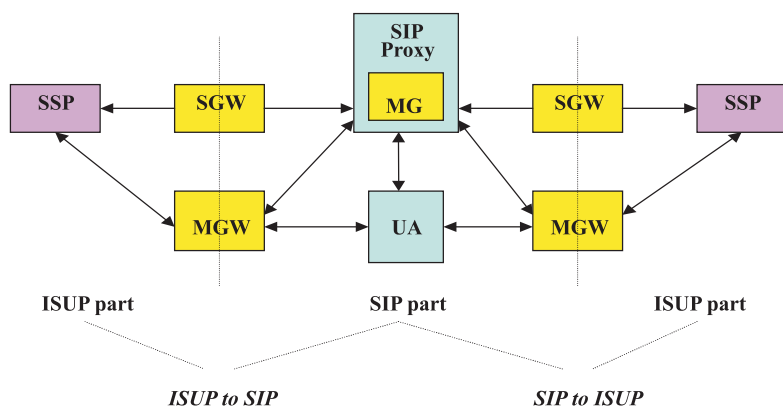

Note: there might be no SGW between the ISUP part and SIP part. In that case it is the MGC that represents the interface between the ISUP and SIP parts.

Within this task, the protocol interworking issues including SIP-ISUP and ISUP-SIP issues were studied as well [1]

\section{Impact of packet loss and jitter to the quality of a voice transmission}

If the transmitted analogue signal has changed its shape for any reason during its transmission over the network, the difference between a received signal and transmitted signal can be assumed as noise. To explain the main idea of this research project part, we will use only the second order characteristic Signal-to-Noise Ratio (SNR), to argue for its use in the network design and management. For simplicity we do not present results for voice, but just for test signal given by the band-limited noise with constant power spectra within this band.

In the sampling theorem the samples are taken regularly, but due to a random delay in the packet switched network the samples are taken irregularly by the reconstruction procedure. The received (reconstructed) signal will be

$$
r(t)=\sum_{k} s(k \Delta) \Phi\left(t-k \Delta-\tau_{k}\right),
$$

Let us suppose that this irregularity expressed by differences (delays) from regular sampling points, creates the point process $\left\{\tau_{k}, k \in Z\right\}$. Let $\left\{\tau_{k}, k \in Z\right\}$ be an ergodic, stationary, random point process with the characteristic function

$$
G(\omega)=E\left[e^{j \omega \tau_{k}}\right], \omega \in \Re, k \in Z .
$$

If $s(k \Delta)$ are pairs of independent variables and test signal has the constant power density then the SNR defined by the ratio of signal average power and noise average power in logarithmic scale

$$
S N R=10 \log \frac{\sigma_{S}^{2}}{\sigma_{N}^{2}},[\mathrm{~dB}]
$$

becomes

$$
S N R=-10 \log 2\left(1-\frac{1}{2 \Omega} \int_{-\Omega}^{\Omega} \operatorname{Re} G(\omega) d \omega\right),[\mathrm{dB}]
$$

Differences between transmitted signal and received signal in which lost samples were replaced by sample approximations, were studied as noise and SNR was used as the objective measure by Jayant and Christensen. More general study of the noise properties, when the lost sample is replaced by a linear combination of other available samples, was obtained in our research. We restrict explanation to the simplest case again. Only zero stuffing (lost sample is replaced by zero) is assumed, and only SNR is used for the noise description. Under these assumptions the noise caused by lost samples (or packets) gives Signal-to-noise ratio expressed in $\mathrm{dB}$

$$
S N R=-10 \log (p),[\mathrm{dB}]
$$

where $p$ is packet (sample) loss probability.

It should be remarked that the analysis of sample loss is easier than the analysis of jitter and using additional noise properties, for example, psofometric weighting is possible.

Until now, the jitter or packet loss was taken as the only source of noise. The approach, in which the impact of the voice distortion sources are given by SNR, allows to calculate the impact of more independent noise sources. Let $n_{1}(t), \ldots, n_{M}(t), t \in \Re$ be independent noises with average powers $\sigma_{N 1}^{2}, \ldots, \sigma_{N M}^{2}$. Then the total signal-to-noise ratio is 
$S N R=10 \log \frac{\sigma_{S}^{2}}{\sum_{i=1}^{M} \sigma_{N i}^{2}}=-10 \log \sum_{i=1}^{M} 10^{-\frac{S N R i}{10}}$,

where $S N R i=10 \log \frac{\sigma_{S}^{2}}{\sigma_{N i}^{2}}, i=1,2, \ldots, M$. As an example the speech

signal is taken with 8-bit $\square$-law ideal quantizing, which produces the noise of $\mathrm{SNR}=38 \mathrm{~dB}$. When this signal is also attacked by the independent jitter with exponential distribution, the total SNR is approximately

$$
S N R \approx-10 \log 2\left(1.000079-\frac{\Delta}{\pi \tau} \operatorname{arctg}-\frac{\pi \bar{\tau}}{\Delta}\right),[\mathrm{dB}] .
$$

A similar function can be obtained for the jitter with uniform and normal distribution, as shown in the following figure.

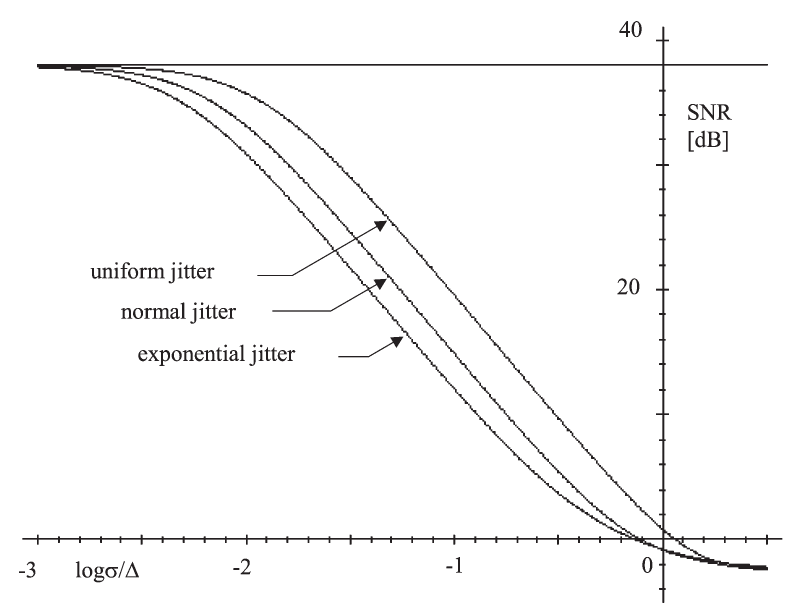

It is recommended for multiplicative noise to use "signal to signal-correlated-noise ratio", called Q when expressed in decibels. Taken into account subjective factors, it is recommended to combine $\mathrm{Q}$ values on a $15 \log 10$ basis, i.e.

$$
Q=-15 \log \sum_{i=1}^{M} 10^{-\frac{Q_{i}}{15}}
$$

In the previous part we assumed that only distortion sources are present and no tool for their compensation is available. These tools are: lost sample/packet replacing techniques and jitter buffer. Lost sample/packet replacing methods are well known from other applications (signal processing, forward error correction codes), but jitter buffer is a new element in telephone network and should be studied carefully. If delay was not a very serious problem in circuit switched telephone networks, compression/decompression, coding/decoding, packetisation and especially waiting in internal network buffers are additional sources of delay. We have no compensator of lost time until now, then it is on the network designers to calculate all delays very carefully.

The total delay occuring in the transmission chain can be divided to two parts: the fixed part of delay and the variable part of the delay - jitter J. If the sum of these delays exceeds a given limit, the delayed packet/sample is discarded by the network i.e. it is lost. To eliminate sample jitter, jitter buffers (play-out buffers) are used. In the case of nonempty jitter buffer, samples are waiting in the queue, and they are played-out in regular intervals. If the buffer is empty when a sample should be sent, a signal gap occurs until the delayed sample is available (no recovery of lost sample is assumed). This residual delay produces again noise, and the previous results may be applied. It is not our aim to study play-out buffers behaviour in its completeness in this contribution. We just take as an example the case, when the delays are independent, i.e. when gaps create a Bernoulli process. Let $p_{0}$ be the probability that a buffer is empty when the sample should be played out, and the distribution of the residual delay i.e. the interval since the sample should be played out is known. Then,

$$
S N R=S N R_{\text {res }}-10 \log p_{0}
$$

This formula shows, how the waiting for the delayed sample improves SNR, compared to the policy when the empty buffer generates zero samples, but just in time. There exist several methods how to improve this result. Firstly, other lost sample replacing methods bring an advantage, but of course, only if their gain in SNR, compared to the zero stuffing is greater than SNRres. Secondly, probability of an empty buffer $p_{0}$ can be decreased if samples are played out slower than the sampling rate, when the buffer is coming to be empty. The static control policy of play-out sample rate can be used, i.e. $\Delta_{n}=\Delta+\delta_{n}, n=1,2, \ldots$ inter-sample interval is used, when the sample should be played out, and the buffer contains n samples. See research reports for details.

In this presentation of the research project we assume static control policy of playout sample rate, i.e. we assume that $\Delta_{n}=$ $=\Delta+\delta_{n}, n=1,2, \ldots$ intersample interval is used, when the sample should be played out, and the buffer contains $n$ samples (infinite buffer capacity is assumed). If the buffer is empty at the playing out instant, the sample will be played out as soon as it comes, i.e. intersample interval $\Delta_{1}+\tau_{\text {res }}$ occurs. The marginal characteristic function of delay is

$$
G(\omega)=E\left[e^{j \omega \tau}\right]=\sum_{n=1}^{\infty} p_{n} e^{j \omega \delta_{n}}+p_{0} E\left[e^{j \omega \tau_{r e s}}\right], \omega \in \Re
$$

and the noise power spectral density, is

$$
\hat{n}(\omega)=2 \sigma_{S}^{2} \hat{\Phi}(\omega)\left[1-p_{0}-\sum_{n=1}^{\infty} p_{n} \cos \omega \delta_{n}\right]+p_{0} \hat{n}(\omega)_{r e s},
$$

$\omega \in \Re$.

The average noise power is

$$
\sigma_{N}^{2}=\frac{2 \sigma_{S}^{2}}{\Delta}\left[1-p_{0}-\sum_{n=1}^{\infty} p_{n} \frac{\sin \pi \epsilon_{n}}{\pi \epsilon_{n}}\right]+p_{0} \sigma_{N \text { res }}^{2},
$$

where $\epsilon_{n}=\frac{\delta_{n}}{\Delta}, n=1,2, \ldots$ is a relative sample delay. The previous formula can be used for SNR calculation. 
ITU-T has proposed a complex method, known as E-model, for voice transmission quality evaluation. All basic network performance parameters of a telephone network are considered. If the voice is transmitted over the packet switched network (IP or ATM), new network performance parameters may increase degradation of the voice quality, the most important being packet loss and rest jitter. There are several approaches to evaluating the impact of packet loss on the transmission quality. Two of them were taken into account. The first one (proposed by ITU-T Rec. G.113 Annex 1) incorporates packet loss to the equipment impairment factor, and the second (described in the paper) incorporates packet loss and rest jitter to the simultaneous impairment factor Is. These models give quite a different evaluation and should be improved. The second approach, used in our research, incorporates packet loss and rest jitter to the simultaneous impairment factor Ie. This approach allowed to combine more degradation factors and respected the fact, that influences of jitter and packet loss are not additive.

\section{Conclusions}

In this contribution we focused on some topical problems of IP Telephony. Having used an example of the "University Infoline: service, we demonstrated the capabilities of new tools for the development of services that advance the service of IP telephony at a qualitatively higher level (SIP and HTTP integration SIP s HTTP). Another problem that is necessary to be solved is interworking of existing network types and terminals with IP-based networks and new types of terminals for IP telephony. In the frame of the Quality of Service (QoS) for IP telephony we proposed analytical models for the noise caused by packed loss and jitter. Influence of packet loss and jitter was incorporated to the E-model introduced by ETSI and ITU-T for an evaluation of voice transmission quality in telephone networks.

\section{References}

[1] N-GOSSIP: Next-Gen open Service Solutions over IP, http://www.eurescom.de/public/projects/P1100-series/P1111/default.asp],

[2] draft-ietf-sigtran-rfc3057bis: http://www.ietf.org

[3] RFC 3331: http://www.ietf.org

[4] draft-ietf-sigtran-m2pa: http://www.ietf.org

[5] RFC 3332: http://www.ietf.org

[6] draft-ietf-sigtran-sua: http://www.ietf.org 